\title{
The Specific Impedance of the Dorsal Columns of Cat: An Anisotropic Medium
}

\author{
James B. Ranck, Jr., and Spencer L. BeMent ${ }^{1}$ \\ Departments of Physiology and Electrical Engineering, University of Michigan, \\ Ann Arbor, Michigan
}

Received October 5, 1964

\begin{abstract}
Low frequency, nonstimulating current was passed from a small electrode on the surface of the dorsal columns in the cervical cord of cats. A glass microelectrode was used to record the voltage at distances of 0.5 to $2 \mathrm{~mm}$ from the current electrode. The voltage fell off more rapidly in depth and across the dorsal columns than it did longitudinally-the resistance was lower in the longitudinal direction. Accordingly, the dorsal columns are anisotropic. An approximate equation is presented which describes the data fairly well and which is consistent with the anatomy. From this equation, the resistivity in the longitudinal direction was 138 to $212 \mathrm{ohm}-\mathrm{cm}$ and in the transverse direction, $1,211 \mathrm{ohm}-\mathrm{cm}$. These values are shown to be consistent with the view that the anisotropy is primarily due to current flowing Iongitudinally in axons. The frequency dependence of the specific impedance was also measured. Some features of this frequency dependence have no clear explanation, but some of them are consistent with a nodal membrane having a time constant of roughly $50 \mu \mathrm{sec}$.
\end{abstract}

\section{Introduction}

The resistance to current flowing longitudinally through the cytoplasm of an axon is always assumed to be much less than the resistance to current flowing transversely across the axon, through the cell membrane, or through myelin. In a peripheral nerve or central nervous system tract of white matter, it would then be expected that the resistance longitudinally would be less than that transversely; i.e., that these tissues would be anisotropic. Rushton cited Hermann (23) as showing that anisotropy exists in a group of peripheral nerves gathered in a bundle, the ratio of resistances being about five to one. In the present study it is shown that anisotropy is also present

1 This investigation was supported by grants NIH NB-04352 from The National Institutes of Health, and by The Information Research Division, Air Force Office of Scientific Research, under Grant AF-AFOSR-367-63. 
in the dorsal columns of cat. However, any interpretation of the result will be complicated by the fact that the status of interstitial space and of nonneuronal current flow in the central nervous system is controversial. Some seem to feel that there is no interstitial space through which current can move, the neuroglia cells being virtually the entire non-neuronal space (10). At the other end of the spectrum are those who seem to feel that the interstitial space is large enough to account for most or all of the non-neuronal current flow (27). We have argued previously (16) that impedance studies cannot distinguish between these possibilities in normal rabbit cerebral cortex. ${ }^{2}$ In this study on current flow in cat dorsal columns, it has not been possible to distinguish between the various possibilities for non-neuronal current flow. However, many aspects of the anisotropy and of the frequency dependence of the specific impedance in dorsal columns can he explained on the basis of commonly accepted principles of neuronal current flow. It therefore will not be necessary to take a stand on non-neuronal current flow.

Cat dorsal columns were chosen for several reasons. They are a large, readily accessible mass of white matter, and they have been studied with the electron microscope by Bunge, Bunge, and Ris (2), who showed that they have a fairly homogeneous fiber distribution, with almost no unmyelinated fibers. Neurophysiologists seem almost always to make the assumption that axons of the central nervous system function similarly to those of the peripheral nervous system (although this assumption is usually implicit). There is little direct evidence for this assumption. Much of the evidence that does exist is from the dorsal columns; specifically, there is evidence of saltatory conduction (25), intracellularly recorded action potentials (11), and studies on the "compound action potential" in vivo and in vitro $(4,5,19,20)$.

\section{Surgical Methods}

Cats were anesthetized with sodium pentobarbital, and tracheotomy and venous cannulation were performed. A partial laminectomy of C-3 and complete laminectomies of $\mathrm{C}-4$ through $\mathrm{C}-7$ were performed with the vertebral column stabilized by clamping onto the spines of C-2 and T-1. The cat was then put on artificial positive pressure respiration. A bilateral pneumothorax was performed and the dura was removed from the dorsal aspect of the cord. Some experiments were performed with the cord under

2 We have not been able to fit the changes in spreading depression except to a view of significant amounts of non-neuronal current flowing both in neuroglia cells and interstitial space (17). 
mineral oil, others with the cord in air. No differences were noted between these two methods. It was not possible to use all parts of the dorsal columns because some parts were covered with a layer of fluid that could not be removed, even by wiping with cotton. Where present, this thin layer of fluid was a sufficiently good conductor to affect the results seriously. No investigation of the cause of the fluid was made. A similar layer has been seen on cat and rabbit cercbral cortex. $\Lambda$ ll the cats had some regions of the surface of the dorsal columns without the fluid layer. All of the experiments were performed on these "dry" parts.

The cord was never exposed to air for more than $15 \mathrm{~min}$., which meant that during some experiments the measurements had to be interrupted and the cord wet with warm saline. At least one pre-wash measurement was then repeated.

Two different experiments were performed. The methods, results, and analysis of each experiment will be given separately, as the rationale and the methods of some of the experiments depend on the results and analysis of others.

\section{Experiment I: Three-Dimensional Low-Frequency Voltage Distribution}

A platinum wire $40 \mu$ in diameter was placed near the midline on the surface of the dorsal columns. Nonstimulating sinusoidal peak current of 5 to $10 \mu a m p$ was passed through this electrode at $5-10 \mathrm{cycle} / \mathrm{sec}$. The cat was grounded through a 470-ohm resistor and a wire in the rectum. The current passed was calculated from voltage measurements across this resistor. The voltage changes in the dorsal columns were measured by a glass microelectrode filled with $3 \mathrm{M} \mathrm{NaCl}$ connected to a cathode follower. These changes were measured against another electrode connected to muscle or bone clamp. The voltage changes in the dorsal columns drove the vertical sweep of an oscilloscope. The voltage across the 470 -ohm resistor drove the horizontal sweep and the resulting pattern was photographed. The position of the microelectrode on the surface of the dorsal column was measured by use of a dissecting microscope with a calibrated eyepiece. The depth of the microelectrode was measured by a calibrated drive on a micromanipulator which held the microelectrode. Four such mappings were made.

We will designate the direction in which the fibers run as the longitudinal direction, the direction normal to the longitudinal direction on the surface of the cord as the crosswise direction, and the direction normal to both of these as the depth (or deep) direction.

The results of one of these experiments is given in Fig. 1. The numbers 
over the dots are proportional to the recorded voltage at that spot. The lines are suggested equipotential lines, drawn free hand from the experimental data.

In all four mappings at a given distance from the current electrode, the voltage was invariably greater in the longitudinal direction than in the

- CROSSWISE -

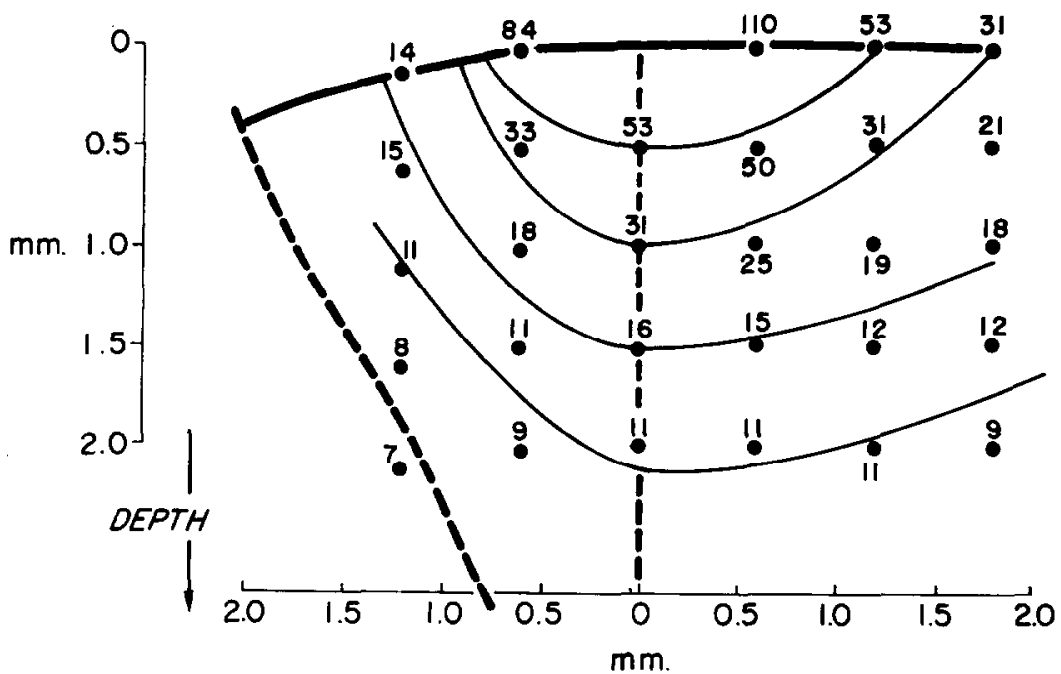

FIG. 1. Three-dimensional voltage distributions from a single small source. The data in all three directions are plotted simultaneously. The plane of the left half of the figure is normal to the plane of the right half. The figure should be folded on the dotted line in the center. The dotted line on the left side indicates the boundary of the dorsal column. The numbers are actually the voltage (in volts) divided by 2.350 times the current passed (in amperes). The current electrode is at the center of the dorsal column at the surface, i.e., where the center dotted line meets the sur ace.

crosswise direction or in depth. The crosswise and depth voltages were not the same, but tended to resemble each other more closely than they resembled the longitudinal voltages. Thus, it is clear that current flows more readily longitudinally than transversely; i.e., the dorsal columns are anisotropic.

The longitudinal and depth voltages fell off approximately as the inverse of the distance from the current source. The decrease of the crosswise voltages with distance was less regular, some falling more rapidly than the inverse of the distance and some less rapidly. The case diverging most from 
the inverse of distance is that shown in Fig. 1. It is not clear why the results in the crosswise direction appear to be so variable. Most of the recordings were taken far enough from the lateral boundary of the dorsal columns so that the resistivity of the surrounding tissue should not have had much influence on the result.

There are at least two factors contributing to the anisotropy. First, in the transverse direction the non-neuronal path curves around the fibers and so will be a maximum of $\pi / 2=1.57$ times longer than in the longitudinal direction where the non-neuronal path is straight and runs in the same direction as the fibers. This effect is independent of the distance from the current electrode. Second, when flowing longitudinally, the current can also flow inside the nerve fibers. This is no doubt the most important factor, as will be seen more clearly below.

Current must flow some distance from the current electrode before it encounters a node of Ranvier where it can enter a fiber. It must flow further yet to encounter another node of the same fiber. Therefore, the effective conductivity which this intracellular path of current flow offers will increase as the distance from the current electrode increases. In the myelinated fibers of frog peripheral nerve, the total resistance between the inside and the outside across the myelin of one internode is about the same as the resistance of one node (26). In the cat dorsal column fibers it might then be expected that some current will enter the fiber across the myelin and will also cause the effective conductivity of the intracellular path to increase with increasing distance from the current source. The glial anatomy is not well enough known to determine whether or not there are neuroglial factors contributing to anisotropy in a way similar to neuronal factors, such as processes extending more extensively in one direction than another. However, any such neuroglial contribution to anisotropy will be much less than any neuronal contribution, because the volume of neuroglia cytoplasm is much smaller than the volume of neuron cytoplasm (2).

Since impedance in each direction should be a function of the distance from the current source, the problem is not only anisotropic, but also nonlinear. We have attempted to solve this system, using a difference equation for current flow in myelinated fibers, but without success. However, the case for anisotropic media with constant impedance in each direction does have a known solution, which will be convenient to use as an approximation. Its use can be justified by the following argument.

The internodal distance in dorsal columns can be estimated from the following data. Bunge, Bunge, and Ris (2) did not make measurements of 
diameters of axis cylinders, but their published electron micrographs of the cervical region of cat dorsal columns show that most of the fibers have axis-cylinder diameters of about $2 \mu$. Häggqvist (8) found a similar distribution for fibers in dorsal column of man. Hess and Young (9) measured the relationship between fiber diameter and internodal length in rabbit spinal cord. They found that the internodal length is a linear function of the fiber diameter, about 170 times the diameter of the axis cylinder (assuming that the axis cylinder is 0.7 of the total fiber diameter). Bodian (1) made similar measurements on several parts of opossum brain and found the internodal length to be 200 times the diameter of the axis cylinder. Therefore almost all fibers will have one node within $200 \mu$ of the current electrode. Even at $500 \mu$ from the current electrode (the shortest distance used in these experiments) many fibers will have two nodes between the current electrode and the recording site. Therefore we are dealing with distances for which the assumption that resistivity is independent of distance from current source may be justified as an approximation, at least as far as the axonal component is concerned. As the distance from the current electrode increases, the assumption will be increasingly valid.

The current flowing in the neuroglia cytoplasm will also contribute to a conductivity which is dependent on the distance from the current electrode. This neuroglial contribution will be small compared to the neuronal contribution in the longitudinal direction because of the relative volume of the neuroglia cytoplasm. In the transverse direction the effect of neuroglial current might be more significant. However, if much current flows through the neuroglia cells as compared to the interstitial space, then the neuroglia cell membrane resistance can be expected to be very low, so that the neuroglial length constant will be much less than $400 \mu$. If the neuroglia membrane resistance and the neuroglia space constant are large, then the interstitial space would be expected to carry most of the non-neuronal current. In either case the distance dependence of non-neuronal transverse conductance can be ignored.

The derivation of the solution of this problem can be found in Maxwell (12) or Rush (21). We are concerned with distances which are large compared to the radius of the current electrode and will consider the surface of the spinal cord flat over the distances used. Therefore

$$
V=I_{0} /(2 \pi)\left(Z_{1} Z_{2} Z_{3}\right)^{1 / 2}\left(Z_{1} x^{2}+Z_{2} y^{2}+Z_{3} z^{2}\right)^{-1 / 2},
$$

where $V$ is voltage; $I_{0}$ is the total current delivered in amperes; $Z_{1}, Z_{2}$, and 
$Z_{3}$ are the specific impedances in ohm-cm in the longitudinal, crosswise, and depth directions respectively; and $x, y$, and $z$ are the distances in $\mathrm{cm}$ from the current electrode in the longitudinal, crosswise, and depth directions, respectively. $\Lambda$ the low frequencies used in this experiment, all the specific impedances are resistivities, as will be shown in Experiment II.

The equation predicts that in the direction of one of the orthogonal axes the voltage falls off as the inverse of distance, a prediction which is satisfied by the experimental results. So, the equation does seem to fit the experimental data fairly well and the assumptions on which it is derived are fairly well met by the dorsal columns. Equation [1] will be used, not because we believe it is particularly accurate quantitatively, but rather to explicate the model being developed and to obtain some order of magnitude values.

From the data of this experiment and Equation [1], the ratio of resistivities can be obtained in two ways. First, on an equipotential in the $x, y$, and $z$ axes, respectively:

$$
\begin{aligned}
V & =I_{0} /(2 \pi)\left(Z_{1} Z_{2} Z_{3}\right)^{1 / 2}\left(Z_{1} x^{2}\right)^{-1 / 2}=I_{0} /(2 \pi)\left(Z_{1} Z_{2} Z_{3}\right)^{1 / 2}\left(Z_{2} y^{2}\right)^{-1 / 2} \\
& =I_{0} /(2 \pi)\left(Z_{1} Z_{2} Z_{3}\right)^{1 / 2}\left(Z_{3} z^{2}\right)^{-1 / 2}
\end{aligned}
$$

so

$$
x / y=\left(Z_{2} / Z_{1}\right)^{1 / 2} \text {, etc. }
$$

That is, on an equipotential, the ratio of the distances from the current source on a Cartesian axis is proportional to the inverse of the square root of the resistivities in those directions. Second, let $V_{r}, V_{y}$, and $V_{z}$ be the values of the voltage at equal distances from the current source on one of the Cartesian axes. Then, from Equation [1]

$$
V_{s} / V_{y}=\left(Z_{1} / Z_{2}\right)^{1 / 2} \text {, etc. }
$$

From the data and Equation [3] or [4], sixteen calculations of the ratio of the square root of the resistivity in the longitudinal direction to the resistivity in the depth direction $\left(Z_{1} / Z_{3}\right)^{1 / 2}$, have been made. $\left(Z_{1} / Z_{3}\right)^{1 / 2}$ is $0.39 \pm 0.02$ (S.E., standard error). So $Z_{1} / Z_{3}=(0.39)^{2}=0.15=1 / 6.7$. The ratio of the square root of the resistivity in the longitudinal direction to that in the crosswise direction $\left(Z_{1} / Z_{2}\right)^{1 / 2}$, from thirteen determinations is $0.53 \pm 0.07$ (S.E.). So $Z_{1} / Z_{3}=(0.53)^{2}=0.28=1 / 3.6$. For all twentynine measurements the ratio was less than one.

From Equation [2], on one of the Cartesian axes,

$$
V=I_{0} /(2 \pi x)\left(Z_{2} Z_{3}\right)^{1 / 2}, \text { etc. }
$$


The voltage difference $(\Delta V)$ between two points on a Cartesian axis $\left(x_{1}\right.$ and $\left.x_{2}\right)$ is

$$
\Delta V=I_{0}\left(Z_{2} Z_{3}\right)^{1 / 2}\left(x_{1}-x_{2}\right) /\left(2 \pi x_{1} x_{2}\right), \text { etc. }
$$

So the voltage or voltage difference on an axis is independent of the resistivity in that direction! This result is one of the shocking properties of current flow in anisotropic media.

From Equation $[5],\left(Z_{2} Z_{3}\right)^{1 / 2}=1,211 \pm 208$ (S.E.) ohm-cm from nine measurements $\left(Z_{1} Z_{\mathrm{s}}\right)^{1 / 2}=408 \pm 41$ (S.E.) ohm-cm from nine measurements; and $\left(Z_{1} Z_{3}\right)^{1 / 2}=507 \pm 56$ (S.E.) ohm-cm from eight measurements. The probability of $\left(Z_{2} Z_{3}\right)^{1 / 2}$ having come from the same population as the other two using the $t$ test is 0.01 or less. The probability of $\left(Z_{1} Z_{2}\right)^{1 / 2}$ and $\left(Z_{1} Z_{3}\right)^{1 / 2}$ having come from the same population is 0.2 by the $t$ test. Therefore let us assume $Z_{2}=Z_{3}$. Then $Z_{2}=Z_{3}=1,211 \mathrm{ohm}-\mathrm{cm}$ and $Z_{1}$ is either 138 or $212 \mathrm{ohm}-\mathrm{cm}$. Thus $Z_{2} / Z_{1}=8.8$ or 5.7 .

\section{Experiment II: Frequency Dependence of the Impedance}

The frequency dependence of the impedance was measured with the electrodes on the surface of the dorsal columns by use of methods previously described (15). Briefly, nonstimulating current was passed from a platinum electrode $40 \mu$ in diameter. The voltage difference was recorded between two similar electrodes also on the surface. The tips of all three electrodes lay on a straight line and were within $1.5 \mathrm{~mm}$ of each other. The animal was grounded through a $470-0 h m$ resistor. The voltage across this resistor was proportional to the current passed and drove the horizontal sweep of an oscilloscope. The recorded voltage difference drove the vertical sweep. The current electrode was placed near the center of the dorsal columns. Measurements were made at 5, 50, 500, 5,000, and 50,000 cycle/ sec. Eight measurements were taken on the longitudinal axis and nine measurements wcre taken on a line through the current electrode in the crosswise direction. Five animals were used.

Figure 2 shows polar plots of the impedance, calculated from Equation [6]. $\Lambda \mathrm{t} 5 \mathrm{cycle} / \mathrm{sec}\left(Z_{1} Z_{3}\right)^{1 / 2}=280 \pm 40$ (S.E.) ohm-cm from eight measurements and $\left(Z_{1} Z_{3}\right)^{1 / 2}=664 \pm 63$ (S.E.) ohm-cm from nine measurements. The probability that these two came from the same population is between 0.02 and 0.01 by the $t$ test. Assuming $Z_{2}=Z_{3}$, then $Z_{2}=664$ ohm-cm, $Z_{1}=217 \mathrm{ohm}-\mathrm{cm}$, and $Z_{2} / Z_{1}=3.0$. The value of $Z_{1}$ is in fair agreement with that determined in Experiment $I$ (138 or $212 \mathrm{ohm}-\mathrm{cm}$ ). The value of $Z_{2}$ does not agree so well with that found from Experiment I 
$(1,211 \mathrm{ohm}-\mathrm{cm})$, but does not differ more than might be expected, in view of the fact that Equation [1] is a crude approximation.

The frequency dependence of the specific impedance was similar in both directions, the maximum phase angle almost always occurring at about 5,000 to $50,000 \mathrm{cycle} / \mathrm{sec}$. Phase angles at frequencies less than 5,000

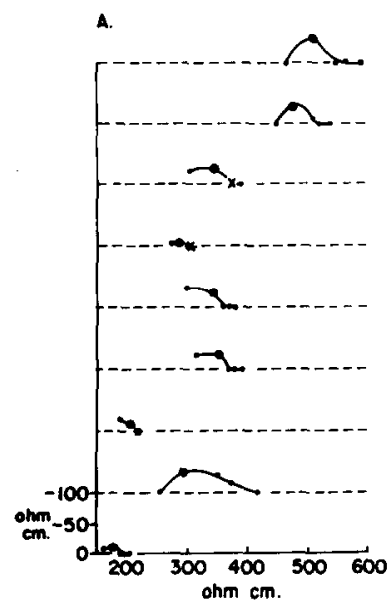

8.

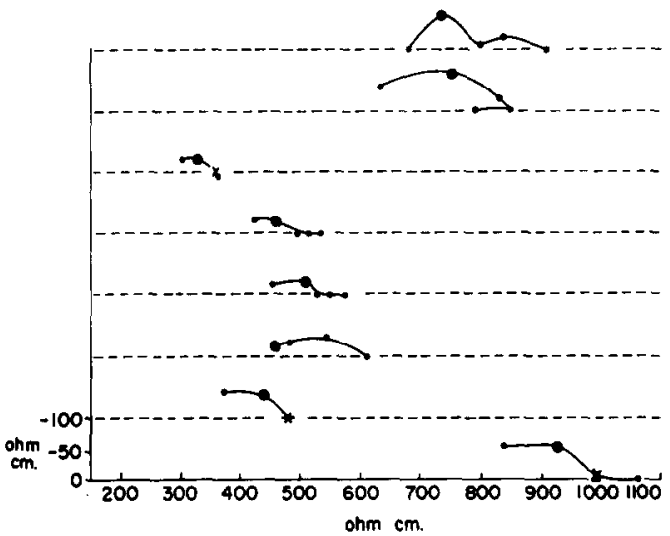

Frc. 2. Polar plots of the impedance. The abscissa is the real part. The ordinate is the imaginary part, with negative up. The origin of all these coordinates is not included, but would be further to the left. The plots in $A$ are of $\left(Z_{1} Z_{3}\right)^{1 / 2}$, i.e., the impedance as measured in the transverse direction. The plots in $B$ are of $\left(Z_{2} Z_{3}\right)^{1 / 2}$, i.e., the impedance as measured in the longitudinal direction. The dots represent measurements at $5,50,500,5,000$, and $50,000 \mathrm{cycle} / \mathrm{sec}$. The large dot is the $5-\mathrm{kc}$ measurement. A cross indicates that the values at two or more frequencies are the same. The line connecting the points was drawn by hand.

cycle/sec are smaller or nonmeasurable. At 5,000 cycle $/ \mathrm{sec}\left(Z_{2} Z_{3}\right)^{1 / 2}=$ $577 \pm 67$ (S.E.) ohm-cm and $\left(Z_{1} Z_{3}\right)^{1 / 2}=331 \pm 34$ (S.E.) ohm-cm. At $50,000 \mathrm{cycle} / \mathrm{sec}\left(Z_{2} Z_{3}\right)^{1 / 2}=532 \pm 59$ (S.E.) ohm-cm and $\left(Z_{1} Z_{3}\right)^{1 / 2}=$ $301 \pm 32$ (S.E.) ohm-cm.

The only well-established mechanism by which phase angles could occur at these frequencies is that of capacitative current flowing across the cell membranes. The major contribution to the current should then be at the nodes where the current flowing longitudinally in the axons is entering or leaving. Therefore, $Z_{1}$ should have a much larger frequency dependence than $Z_{2}$ and $Z_{3}$. From Equation [1] above, it has been shown that the impedance measurements in the longitudinal direction are independent of 
the impedance in that direction. The fact that phase angles of similar magnitude were seen in both directions may be due to the inadequacy of Equation [1] or to the fact that there is appreciable glial cytoplasmic current in the transverse direction or both. Schwan (24) has suggested that there may be a frequency dependent impedance in biological tissue which is the result of peculiarities of the surface conductance. Fatt (6) has discussed this mechanism and concluded that it is unlikely that it is of much importance for cases such as we are considering. The basis for the apparent frequency dependence of $Z_{2}$ and $Z_{3}$ is not clear.

We have not been able to obtain a solution for current flow in myelinated fibers for this three-dimensional case. Physical intuition suggests that the membrane time constant of the nodal fibers will be of the order of the inverse of the frequency of greatest phase angle, i.e., about 200-20 $\mu$ sec. $^{3}$ The time constant of a node of Ranvier of a frog sciatic nerve is about 50 usec (26). Nodes of the dorsal column would seem to have values similar to this.

\section{Discussion}

Anisolropy. Some of the peculiarities of anisotropic current flow have already been mentioned. We have found that our intuitions are very poor and deceptive with regard to anisotropy. Many of the usual relationships do not hold. For example, lines of current flow are not normal to the equipotential lines, and Laplace's equation does not hold.

Rush (21) and Rush, Abildskov, and McFee (22) have also discussed anisotropic current flow in biological material. Anisotropy can be expected to occur in the central nervous system wherever fibers run predominantly in one direction. It should be considered in recording, stimulating, lesion making, and diffusion. In many cases, much smaller distances than those used in this study are important, and the additional complication of distance dependent conductivities must be considered.

Specific Impedance of White Matter. In the present study, numerical values of the specific impedance of the dorsal columns have been determined only approximately. However, it is clear that the specific impedance in the transverse direction in the dorsal columns is several times higher than that of rabbit and cat cerebral cortex, which is about $250 \mathrm{ohm}-\mathrm{cm}$ (at $1,000 \mathrm{cycle} / \mathrm{sec})(7,15,28)$. The specific impedance of the dorsal

3 Patlack's analysis (13) of the frequency dependence of current flow in a myelinated fiber for the one-dimensional case is a relevant and useful aid to this intuition. 
columns in the longitudinal direction is similar to or lower than that in the cerebral cortex. The specific impedance of rabbit subcortical white matter, measured by Van Harreveld, Murphy, and Nobel (28), is $960 \mathrm{ohm}-\mathrm{cm}$ or 4.6 times that of cortex. Freygang and Landau (7) reported the specific impedance of cat subcortical white matter to be about 1.5 times that of cortex, or $333 \mathrm{ohm}-\mathrm{cm}$. Robinson's (18) results also imply that white matter generally has a distinctly higher impedance than gray matter. The frequency dependence of the impedance is also distinctly different from that of cerebral cortex.

If the transverse current flows purely in an interstitial space with a resistivity like that of cerebrospinal fluid $(60 \mathrm{ohm}-\mathrm{cm}$, an "equivalent conductive interstitial space"), then $1,211 \mathrm{ohm}-\mathrm{cm}$ is equivalent to a $13 \%$ equivalent conductive interstitial space and $664 \mathrm{ohm}-\mathrm{cm}$ to a $7 \%$ space. Davson, Kleeman, and Levin (3) found a 10\% PAH and 12\% sucrose space for whole spinal cord after equilibration with cerebrospinal fluid, so these values determined from impedance data seem plausible. If, however, neuroglia cells carry any transverse current, this space is correspondingly less.

The conductivity in the longitudinal direction is due largely to current flow in axoplasm, with no geometrical impediments to flow. Since the axis cylinder is about 0.7 of the total diameter of a fiber, it is 0.49 of the volume of the fiber, and only slightly less than this of the whole volume of the dorsal columns. Values of the longitudinal resistivity obtained (138, 212 , and 217 ) seem reasonable, since the resistivity of cytoplasm is 1.5 to 3 times that of interstitial fluid in all cells studied (14).

\section{References}

1. Bodian, D. 1951. A note on nodes of Ranvier in the central nervous system. J. Comp. Neurol. 94: 475-483.

2. Bunge, M. B., R. P. Bunge, and H. Ris. 1960. Electron microscopic study of demyelination in an experimentally induced lesion in adult cat spinal cord. J. Biophys. Biochem. Cytol. 7: 685-696.

3. Davson, H., C. R. Kleeman, and E. Levin. 1962. Quantitative studies of the passage of different substances out of the cerebrospinal fluid. J. Physiol. London 161: $126-142$.

4. Eisenman, G., and D. O. Rudin. 1954. Further studies on the functional properties of spinal axons in vivo. J. Gen. Physiol. 37: 495-503.

5. Eisenman, G., and D. O. Rudin. 1954. The compound origin of potential in a stimulated dorsal root. J. Gen. Physiol. 37: 781-793.

6. FatT, P. 1964. An analysis of the transverse electrical impedance of striated muscle. Proc. Roy. Soc. London, Ser. B. 159: 606-651. 
7. Freygang, W. H., and W. M. Landau. 1955. Some relations between resistivity and electrical activity in the cerebral cortex of the cat. J. Cell. Comp. Physiol. 45: $377-392$.

8. HäGGQvist, G. 1936. Analyse der Faserverteilung in einem Ruckenmarkquerschnitt (Th 3). Z. Mikrosk. Anat. Forsch. 39: 1-34.

9. Hess, A., and J. Z. Young. 1949. Correlation of internodal length and fibre diameter in the central nervous system. Nature 164: 490-491.

10. Karlsson, U, and R. Schultz. 1964. Plasma membrane apposition in the central nervous system after aldehyde perfusion. Nature 201: 1230.

11. KoKetsu, K. 1956. Intracellular potential changes of primary afferent nerve fibers in spinal cords of cats. $J$. Neurophysiol. 19: 375-392.

12. Maxwell, J. C. 1891. "A Treatise on Electricity and Magnetism," 3rd ed., Sect. 297-302. Dover, New York.

13. Patlack, C. S. 1955. Potential and current distribution in nerve: The effect of the nerve sheath, the number of fibers, and the frequency of alternating current stimulation. Bull. Math. Biophys, 17: 287-307.

14. Pauly, H., and L. Packer. 1960. The relationship of internal conductance and membrane capacity to mitochondrial volume. J. Biophys. Biochem. Cytol. 7: 603-612.

15. Ranck, J. B, JR. 1963. Specific impedance of rabbit cerebral cortex. Exptl. Neurol. 7: 144-152.

16. Ranck, J. B., JR. 1963. Analysis of spccific impedance of rabbit cercbral cortex. Exptl. Neurol. 7: 153-174.

17. Ranck, J. B., Jr. 1964. Specific impedance of cerebral cortex during spreading depression, and an analysis of neuronal, neuroglial, and interstitial contributions. Exptl. Neurol. 9: 1-16.

18. Robinson, B. 1962. Localization of intracerebral electrodes. Exptl. Neurol. 6: 201-223.

19. Rudin, D. O., and Eisenman, G. 1953. After potential of spinal axons in vivo. J. Gen. Physiol. 36: 643-657.

20. Rudin, D. O., and Eisenman, G. 1954. The action potential of spinal axons in vitro. J. Gen. Physiol. 37: 505-538.

21. Rush, S. 1962. Methods of measuring the resistivities of anisotropic conducting media in situ. J. Research Natl. Bur. Stand. C 66: 217-222.

22. Rush, S., J. A. AblLdskov, and R. MCFEe. 1963. Resistivity of body tissues at low frequencies. Circulation Research 12: 40-50.

23. Rushton, W. A. H. 1927. Effect upon the threshold for nervous excitation of the length of nerve exposed and the angle between current and nerve. J. Physiol. London 63: $357-377$

24. Schwan, H. P. 1957. Electrical properties of tissue and cell suspensions, pp. 148-209. In "Advances in Biological and Medical Physics." J. H. Lawrence and C. A. Tobias [eds.]. Academic Press, New York.

25. TASAKI, I. 1952. Properties of myelinated fibers in frog sciatic nerve and in spinal cord as examined with micro-electrodes. Japan. J. Physiol. 3: 73-94.

26. TASAKI, I. 1955. New measurements of the capacity and the resistance of the 
myelin sheath and the nodal membrane of the isolated frog nerve fiber. $A \mathrm{~m}$. J. Physiol. 181: 639-650.

27. Van Harreveld, A, and J. Crowell. 1964. Extracellular space in central nervous tissue. Federation Proc. 23: 304.

28. Van Harreveld, A., T. Murphy, and K. W. Nobel. 1963. Specific impedance of rabbit's cortical tissue. Am. J. Physiol. 205: 203-207.

NOTE ADDED IN PROOF

Recently I. Tasaki (1964. A New Measurement of Action Currents Developed by Singular Nodes of Raniver. J. Neurophysiol. 27: 1199-1206.), has shown that the specific resistance of toad sciatic nerve is 50 times larger in the transverse than in the longitudinal direction. 